\title{
Unterwanderung des Jugendschutzes und Verletzung der WHO-Rahmenkonvention
}

\section{Rainer M. Kaelin}

Facharzt für Innere Medizin und Pneumologie, vormals Vizepräsident der Lungenliga Schweiz und der Lungenliga Waadt
Dass Tabakprodukte, deren Vertrieb, Promotion, Werbung und Sponsoring einer Gesetzgebung bedürfen, steht ausser Frage. Das weltweit durch Tabak verursachte immer noch wachsende Public-HealthProblem lässt daran keine Zweifel offen. Die den Tabak betreffenden Bestimmungen sind zurzeit im Lebensmittel(!)-Gesetz vereint. Sie sollen in einem eigenen Bundesgesetz vereint werde. Nachdem ein solches Tabakproduktegesetz (TabPG) angenommen sein wird, wird das Bundesparlament auch die Tabakrahmenkonvention der WHO ratifizieren können.

Der am 21.5.2014 von Bundesrat Alain Berset vorgestellte Entwurf zum Tabakproduktegesetz erklärt als sein Ziel: die Jugendlichen sollen geschützt werden. Sie sind vor allem gefährdet. Die meisten abhängigen Raucher/-innen beginnen vor dem zwanzigsten Lebensjahr mit ihrem Abhängigkeitsverhalten und es sind diese Früheinsteiger/-innen, die sich am schwersten tun, das Rauchen aufzugeben. Logisch, dass der Verkauf von Tabak an Jugendliche verboten sein soll; auch Werbung auf Plakaten, im Kino, in Printmedien und auf elektronischen Trägern soll verboten sein, da sie «grossen Einfluss (ausübt) und Anreize schafft». Tatsächlich ist erwiesen, dass umfassende Werbe- und Sponsoringverbote entscheidend zur Denormalisierung des Rauchens in der Gesellschaft beitragen und dadurch den Einstieg erschweren.

\section{«Der Gesetzesentwurf erfüllt klar seinen Zweck nicht.»}

Unverständlich ist daher, wenn das Sponsoringverbot nur für internationale Anlässe gelten und die Werbung an Verkaufsstellen und auf «Produkten, die einen direkten Bezug zum Tabak haben», weiterhin erlaubt sein soll. Das heisst also, dass weiterhin Zigarettenautomaten in Gaststuben, Aschenbecher auf Terrassen, Plakatwerbung und Beschriftungen von Fumoirs und Raucherbeizen und Banalisationsoberflächen hinter den Kassen der ubiquitär rund um die Uhr offenen Shops und der Kioske für genau das sorgen, was das Gesetz zu vermeiden vorgibt. Die mit untrüglichem Gespür für zweideutige Botschaften begabten Jungen werden dadurch das Rauchen weiterhin als banal wahrnehmen. Und die «nationalen Anlässe», an denen Sponsoring durch Tabakfirmen zugelassen sein soll, werden das ihre dazu beitragen, die Ambivalenz der sogenannten Präventionsbotschaft zu verstärken. Angehende Fussballer, die durch ihre Trainer während der Woche zum rauchfreien Sport angehalten worden sind, dürfen dann beim Match mit dem Zigarettenlogo des Sponsors auftreten. Ausserdem - wie wird man einen «nationalen» von einem «internationalen Anlass» unterscheiden können? Ist beispielsweise ein von Japan Tobacco gesponsertes Grümpelturnier oder ein Open-Air-Konzert im Kanton Luzern mit internationalem Star ein nationaler Anlass? Oder gilt dies erst, wenn eine Gruppe von Sportlern oder Musikern aus dem Elsass daran teilnimmt? Man darf vermuten, dass die internationalen Anlässe Erwachsene ansprechen werden (die als Zielpublikum nicht interessieren). In einer Konsumgesellschaft, in der planetäre Wirtschaftsakteure Information, Desinformation, Werbung und Promotion abgestimmt auf lokale und nationale Gegebenheiten orchestrieren, garantieren Ausnahmen dieser Art die Unwirksamkeit der vom Bundesrat vorgeschlagenen Massnahme.

Dass im Gesetzesentwurf die E-Zigaretten den Tabakzigaretten gleichgestellt werden, erscheint folgerichtig. Dass aber das neue Tabakproduktegesetz nur für nikotinhaltige E-Zigaretten gelten soll, macht das Werbeverbot unwirksam und unglaubwürdig. Einerseits gebrauchen die meistens «Dampfer» nikotinhaltige Liquids, aber weder im Gebrauch, noch im Aspekt kann man dies von der Verwendung nikotinfreien Liquids unterscheiden. Andererseits verwendet die Industrie diese Geräte, wie auch die Nargilehs, Shishas usw. schon jetzt gezielt als Promotionsund Marketinginstrument für die Jungen. Wir dürfen uns darauf gefasst machen: Das seit langem geltende Werbeverbot an Radio und Fernsehen und das vorgesehene Werbeverbot in andern Medien wird munter mit Werbespots umgangen werden, in denen dampfende Jugendliche ihre Altersgenossen auffordern, doch auch das modische Spielzeug zu versuchen. Wobei diskret darauf hingewiesen wird, dass es sich - natürlich - um nikotinfreie E-Zigaretten handelt. Diese Art der Täuschung wird übrigens jetzt schon in der Fernsehwerbung für alkoholfreies Bier verwendet! Der Gesetzesentwurf ignoriert hierbei die ihr zugedachte Rolle der E-Zigarette, das Rau-
Dr. med. Rainer M. Kaelin

2, place Hôtel de Ville CH-1110 Morges

rmkaelin[at]sunrise.ch 
chen in der Gesellschaft und im heutigen audiovisuellen Umfeld zu renormalisieren.

Bewusst, dass eine solche Gesetzgebung unbefriedigend ist, bemäntelt der Bundesrat die erwähnten Halbmassnahmen mit dem Ausdruck «moderate Bestimmungen». Dennoch erscheint es klar, dass unsere Behörden dem Druck der Zigarettenhersteller nachgegeben haben. So untergraben die Ausnahmen das vorgegebene Ziel des Gesetzes, den Jugendschutz. Der Gesetzesentwurf erfüllt klar seinen Zweck nicht. Die Bundesbehörden schicken sich erneut an, die Öffentlichkeit zu täuschen, wie dies mit dem Alibi-Bundesgesetz zum Passivrauchschutz [1] der Fall war. Die Raucherzahlen stagnieren in der Schweiz seit einigen Jahren um die $27 \%$. Das ist rund doppelt soviel als in Australien, wo die strukturellen Massnahmen konsequent auf die Denormalisierung des Rauchens hinzielen, (ohne Tabakprodukte oder das Rauchen zu verbieten). Hinter den Zahlen verbirgt sich leider die beunruhigende Tatsache, dass der Anteil der jungen Raucher/innen in der Schweiz steigende Tendenz zeigt. Offenbar waren Marketing-, Promotions- und Sponsoringinvestitionen der Industrie in den letzen Jahren erfolgreich. Und dies genau in dem Alterssegment, in dem die Prävention den nachhaltigsten Erfolg hätte. «Die für die Industrie erträglichen Bestimmungen» des Gesetzesentwurfes erscheinen wie ein an diese gerichteter Applaus, die derzeitigen Präventionsbemühungen des Staates erfolgreich umgangen zu haben.

Schon vor zehn Jahren hat der damalige Gesundheitsminister Pascal Couchepin die Rahmenkonvention der Weltgesundheitsorganisation zur Kontrolle des Tabaks unterschrieben. Dieses von den meisten Ländern der Welt auch ratifizierte Vertragswerk (die Schweiz mit wenigen andern bleibt eine Ausnahme) verpflichtet die Parteien, nicht nur das tödliche Produkt Tabak, sondern auch das Verhalten der Industrie, die das Problem der öffentlichen Gesundheit verursacht, in Schranken zu halten. Die Konvention hält fest, dass das Rechtsgut Gesundheit gegenüber wirtschaftlichen Partikularinteressen Vorrang hat. Denn «der Tabakkonsum mit seinem Gefolge von Toten ist das Paradigma einer industriellen Epidemie, verursacht durch die Profitgier von (...) transnationalen Firmen, auf Kosten der Gesundheit der Bürger und der Staatsrechnungen» [2]. Den kantonalen Gesundheitsdirektionen, den Bundesbehörden und den Parlamentariern, die das TabPG erlassen werden, sei in Erinnerung gerufen: Seit Jahrhunderten bekämpft man Epidemien mit Massnahmen, die Verbreitung und Bewegungsfreiheit der Vektoren hin- dern oder unterbrechen. Ein zahnloses Tabakproduktegesetz könnte zu einer perversen Situation führen: Das Bundesparlament ratifiziert die Rahmenkonvention zur Tabakkontrolle der WHO unter Missachtung ihres erklärten Zieles und Inhaltes.

Ärzte-, Gesundheits- und Präventionsorganisationen und die Medien sind aufgerufen, durch Demaskierung von Desinformation und Überzeugungsarbeit, die auf wissenschaftliche Evidenz basiert, auf eine Gesellschaft hinzuarbeiten, in der Rauchen nicht mehr die Norm ist. Den Entwurf des TabPG als «einen Schritt in die richtige Richtung» zu bezeichnen, ist unehrlich. Die Schweizer Bevölkerung hat ein Recht auf wahrheitsgemässe Information. Dies besonders, wenn es um das Verführen unserer Kinder durch das Sponsoring einer Industrie geht, die sich nie einen Deut um das Gesamtwohl der Gesellschaft gekümmert hat.

Der Vorstand von «Hausärzte Schweiz» steht hinter den Aussagen dieses Artikels; seine Stossrichtung deckt sich mit der Vernehmlassungsposition der Berufsorganisation.

Mitunterzeichner:

Prof. émér. Jean-Claude Chevrolet, ancien médecin chef des soins intensifs HCUGE, Genève;

Dr. med. Gerhard Ernst, Facharzt für Allgemeinmedizin, Himmelried

Dr. med. Hanspeter Faes, Facharzt für Allgemeinmedizin, Ärzte-Gesundheitszentrum Lägern, Ehrendingen; Dr. med. Alexander Möller PD, Leitender Arzt Pneumologie Kinderspital Zürich;

Dr Reto Olgiati, Médecine interne et Pneumologie, Délémont;

Dr. med. Helmut Oswald, Leitender Arzt Pneumologie Kantonspital Winterthur;

Dr. med. Erich Stritt, Facharzt für Kinder und Jugendliche, Belegarzt Neonatologie Dalerspital, Schularzt, Düdingen; Prof. émér. Jean-Marie Tschopp. Ancien médecin chef Centre valaisan de Pneumologie, Montana;

Dr Christophe Uldry, Pneumologie, médecin chef Hôpital de Rolle;

Dr. med. Alice Zürcher, Pneumologie, Oberärztin Spital Uster;

Dr Jean Claude Zellweger,PD, Pneumologie, Fribourg

\section{Literatur}

1 Kaelin RM. Prävention und Glaubwürdigkeit von Gesundheitspolitikern: Ein Minenfeld. Schweiz Ärztezeitung. 2012;93(23):873-7.

2 Bulletin épidémiologique hebdomadaire français. Mai 2013. 